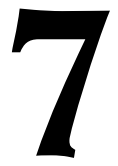

\title{
CONSUMO DE DROGAS EN ADOLESCENTES ESCOLARIZADOS INFRACTORES
}

(DRUG USE IN SCHOOLED ADOLESCENT OFFENDERS)

José-María Faílde Garrido

María D. Dapía Conde

Alberto Alonso Álvarez

Eliézer Pazos Millán

Universidad de Vigo

DOI: $10.5944 / e d u c X X 1.14600$

Cómo referenciar este artículo/How to reference this article:

Faílde Garrido, J. M.; Dapía Conde, M. D.; Alonso Álvarez, A. y Pazos Millán, E. (2015). Consumo de drogas en adolescentes escolarizados infractores. Educación XX1, 18(2), 167-188, doi: 10.5944/ educXX1.14600

Faílde Garrido, J. M.; Dapía Conde, M. D.; Alonso Álvarez, A. \& Pazos Millán, E. (2015). Consumo de drogas en adolescentes escolarizados infractores [Drug use in schooled adolescent offenders]. Educación XX1, 18(2), 167-188, doi: 10.5944/educXX1.14600

\section{RESUMEN}

En las últimas décadas ha habido un interés creciente en la investigación acerca del consumo de drogas en los adolescentes. Sin embargo, es muy poco lo que conocemos sobre el consumo de sustancias en adolescentes infractores. Este estudio tiene como objetivo describir los patrones de consumo de drogas en adolescentes infractores de Galicia escolarizados en $4 .^{\circ}$ de Educación Secundaria Obligatoria (ESO) y compararlos con los de estudiantes no infractores de su mismo nivel educativo y edad. La muestra total fue de 491 participantes, de los cuales 92 eran adolescentes infractores y 399 adolescentes no infractores. Los resultados indican diferencias estadísticamente significativas en el consumo de sustancia entre ambos grupos, siendo más elevado en los adolescentes infractores. Asimismo, se han detectado diferencias significativas en el porcentaje de policonsumidores, en el número de sustancias consumidas y en la edad de inicio de consumo de sustancias. En conclusión, los resultados ponen en evidencia la necesidad de intervenciones educativas dirigidas a prevenir el consumo de drogas en este colectivo. 


\section{PALABRAS CLAVE}

Adolescentes; jóvenes infractores; uso de drogas; justicia juvenil; epidemiología; estudiantes.

\section{ABSTRACT}

In recent decades there has been a growing interest in research on drug use in adolescents. However, there is little we know about substance use in adolescent offenders. This study aims to describe patterns of drug use in Galician adolescents offenders, schooled in the 4th year of Secondary Education (ESO), and compare them to adolescents of the same educational level and age. The total sample consisted of 491 participants, of whom 92 were adolescents in situations of social conflict and 399 were community adolescents. The results indicate statistically significant differences in substance abuse among both groups, with higher incidence in adolescent offenders. Significant differences have also been detected in the percentage of polyconsumers, the number of consumed substances and the age of onset for some substances. In conclusion the results highlight the need for educative and preventive interventions aimed at this group.

\section{KEY WORDS}

Adolescents; juvenile offenders; drug use; juvenile justice; epidemiology; students.

\section{INTRODUCCIÓN}

La adolescencia es una etapa de suma importancia en el desarrollo del ser humano, marcada por cambios a nivel emocional, psicológico y social, que influyen en su comportamiento (Moreno, 1998). Es en esta fase evolutiva en la que suelen iniciarse las salidas sin compañía de los adultos y en la que el grupo de iguales ejerce un papel fundamental en la construcción de la identidad. En este contexto los y las adolescentes ven facilitado el acceso a sustancias como el alcohol, el tabaco, el cannabis, y además es cuando se inician, en un porcentaje importante, en los consumos de diferentes sustancias adictivas (Espada, Méndez, Griffin y Botvin, 2003).

Al igual que en años anteriores, en la Encuesta estatal sobre uso de drogas en estudiantes de enseñanzas secundarias (ESTUDES), 2010- las drogas más consumidas han sido el alcohol, el tabaco y el cannabis: un 75,1\% refiere haber tomado bebidas alcohólicas alguna vez en su vida, un 39,8\% 
tabaco y un 33\% cannabis; reflejándose, no obstante, un descenso en el consumo de estas sustancias respecto a años anteriores (DGPNSD, 2011). Si bien el consumo de otras sustancias presenta menor prevalencia, no debe distraernos de las investigaciones que relacionan el consumo de alcohol y tabaco como la puerta de acceso para iniciarse en el consumo de otras drogas ilegales (Kandel y Davies, 1992; Lindsay y Rainey, 1997).

El consumo de drogas en la etapa adolescente constituye, en las últimas décadas, un foco de interés creciente en las investigaciones. Algunas de las conclusiones de estos estudios apuntan hacia un inicio cada vez más temprano del consumo, cambios en los modos de consumir, así como una tendencia a la igualación de los patrones de consumo entre chicos y chicas (Espada et al., 2003; DGPNSD, 2011; Mendoza, López y Sagrera, 2007).

Según la legislación española, Ley 3/2011 de 30 de junio de apoyo a la familia y la convivencia en Galicia, se entiende por menor infractor aquel o aquella que hubiera cometido hechos tipificados como delitos o faltas en el código penal o en las leyes penales especiales (mayores de 14 y menores de 18 años) en virtud de lo dispuesto en la Ley Orgánica 5/2000 Reguladora de Responsabilidad Penal del Menor.

Sin embargo, este concepto no puede ser literalmente aplicable al ámbito internacional, lo que dificulta el análisis comparativo. No obstante, entre los estudios publicados fuera de España, podemos citar la investigación realizada por Sáenz, Bejarano y Fonseca (2006) con 91 jóvenes infractores en Costa Rica, en la que se concluye que el consumo de sustancias tanto legales como ilegales se inicia a edades muy tempranas, su prevalencia es muy elevada y significativamente mayor que el reportado por la población general. Por su parte, CONACE (2002), en otro estudio desarrollado en Chile corrobora que las tasas de consumo de drogas fueron considerablemente más altas entre los menores infractores en comparación con los adolescentes escolarizados. En una misma línea, un estudio realizado en Río de Janeiro (Brasil), reporta niveles muy elevados de consumo entre la población de adolescentes infractores de la ley (Andrade, Silva y Assumpção, 2004). Más recientemente en un estudio realizado por el Instituto Colombiano de Bienestar Familiar (ICBF) y la Dirección Nacional de Estupefacientes (DNE) del Gobierno Nacional de Colombia sobre el consumo de sustancias en adolescentes en conflicto con la ley, se concluye que el consumo de sustancias en adolescentes infractores es muy superior al observado en la población general; siendo las sustancias ilegales más consumidas la marihuana, los sedantes, la cocaína y los inhalables (ICBF/ DNE, 2010). Resultados similares han sido informados en otros estudios internacionales (Instituto de Sociología UC, 2007; Gobierno Nacional de la República de Colombia, 2009; UNODC, 2010). 
Por otra parte, es muy poco lo que conocemos en nuestro medio sobre el consumo de sustancias de los menores que se hallan en los centros de protección y reeducación, y en particular de estos últimos donde se cumplen medidas privativas de libertad. Tras una exhaustiva búsqueda en las principales bases de datos científicas (PsycInfo, Scopus, The Web of Science y Medline) y en los diferentes recursos que integra el motor de búsqueda Bugalicia (Consocio de Bibliotecas de las universidades gallegas), podemos concluir que en España son escasas las publicaciones disponibles sobre el consumo de drogas en adolescentes infractores. De los cinco estudios que hemos localizado, tres son memorias de proyectos de investigación (DíazAguado, Martínez y Martín, 2002; Esteban, Díaz y Gañan, 2003; Gamonal, Insua y Moreno, 2009) y dos son estudios retrospectivos que se basan en los análisis de los expedientes de menores infractores (Contreras, Molina y Cano, 2012; San Juan, Ocáriz y Germán, 2009). Este hecho contrasta con la consideración de alta vulnerabilidad de este colectivo hacia el uso y abuso de drogas, tal como se recoge en la Estrategia Nacional sobre Drogas 20092016 (Ministerio de Sanidad y Política Social, 2009) y por el Observatorio Europeo de las Drogas y las Toxicomanías (2003). El primero de los estudios citados fue realizado en centros de menores de la Comunidad de Madrid (Díaz-Aguado, Martínez y Martín, 2002) y en sus conclusiones se indica que las sustancias ilegales más consumidas fueron los inhalables, los alucinógenos y la heroína; presentando, por tanto, un patrón de consumo diferente al resto de la población adolescente, en el cual las sustancias ilegales más consumidas suelen ser el cannabis, los hipnosedantes y la cocaína (DGPNSD, 2011); además también señala que el inicio del consumo de drogas en este colectivo es más precoz que en el resto de la población. Por otra parte, en el año 2002, la Delegación del Gobierno para el Plan Nacional sobre Drogas subvencionó el estudio «Menores infractores con consumo de drogas en los centros de reforma» (Gamonal, Insua y Moreno, 2009), llevado a cabo en 26 centros de ocho comunidades autónomas de España, el cual señala entre sus resultados la temprana edad de inicio en el consumo de drogas por parte de estos jóvenes y la alta prevalencia de consumo de hachís y estimulantes. Dos años más tarde, la misma Delegación subvencionó otro estudio titulado "Análisis de la situación de los centros de protección y reforma en el ámbito de la prevención», realizado en 23 centros de cuatro comunidades autónomas (Contreras, Molina y Cano, 2012), cuyos resultados revelan que en este colectivo los consumos de drogas son muy superiores a la población general de la misma edad, siendo mayor en centros de reforma que de protección y con un consumo claramente diferenciado entre mujeres y hombres. Por otra parte, los estudios realizados a partir de los análisis de expedientes de adolescentes infractores evidencian, también, prevalencias elevadas e inicios significativamente más tempranos que la población adolescente no infractora (Ministerio de Sanidad y Política Social, 2009; San Juan, Ocáriz y Germán, 2009). 
En este contexto, el objetivo de esta investigación pretende describir los patrones de consumo de drogas en adolescentes infractores gallegos escolarizados en cuarto de la ESO y compararlos con los de estudiantes no infractores de su mismo nivel educativo y edad.

\section{MÉTODO}

\section{Participantes}

La población objeto de estudio la constituyen la totalidad de adolescentes infractores, que ejecutaban medidas judiciales en entidades pertenecientes al sistema de reeducación de la comunidad autónoma de Galicia, compuesta por 174 chicas y chicos. Teniendo en cuenta el reducido tamaño de la población, se optó por seleccionar a todos/as los/as adolescentes que estaban escolarizados en el curso 2011-2012 en cuarto de la ESO, resultando una muestra de 92 participantes, de los que el 67,4\% eran chicos y el 32,6\% chicas, con una edad media de $15,95(\mathrm{Sx}=, 95)$ y un rango situado entre los 14 y los 17 años.

Con efectos de contrastación empírica, fue seleccionada una segunda muestra de adolescentes sin medidas judiciales, que hemos denominado «no infractores», y que también cursaban cuarto de la ESO en el curso 2011-2012. Esta estaba integrada por 399 participantes, de los que el 68,8\% eran chicos y el $33,2 \%$ chicas, con una edad media de $15,83(\mathrm{~S} x=, 84)$ y un rango de 14 a 17 años.

Ambas muestras resultaron equivalentes en relación a la edad $(\mathrm{F}=1,44$, $\mathrm{p}=, 231)$ y a la distribución por género $\left(\mathrm{X}^{2}=, 01, \mathrm{p}=, 918\right)$.

\section{Instrumentos}

Para la recogida de datos se administró un cuestionario, diseñado ad hoc, que incluía una sección relativa al consumo de sustancias (sustancias consumidas - alguna vez y en el último mes-, edades de inicio, frecuencia y patrones de consumo). En su diseño, con el objeto de facilitar la comparación con estudios nacionales relevantes, se han incluido cuestiones extraídas y/o adaptadas de la Encuesta del Plan Nacional sobre Drogas y del Plan Gallego sobre Drogas. Por lo que se refiere a los aspectos sociodemográficos se incluían cuestiones referidas a edad, género, historial académico, rendimiento académico (número de asignaturas pendientes del curso anterior) y estudios de los padres. La versión resultante del cuestionario diseñado ha sido validada mediante la técnica Delphi; en concreto fue sometido al juicio de cinco jueces expertos de distintos ámbitos profesionales (medicina, 
educación y psicología). Asimismo, fue aplicado a una muestra piloto de estudiantes de secundaria.

\section{Procedimiento}

Para la selección de los participantes de la muestra de adolescentes infractores, en primer lugar se solicitó autorización a la Consellería de Familia del Gobierno gallego, quien nos proporcionó un listado de todos los centros de menores de Galicia. De una población total de 174 adolescentes se seleccionó a todos los que estaban cursando $4 .^{\circ}$ de la ESO, resultando un total de 92 participantes.

Por otra parte, se seleccionó una muestra representativa de jóvenes no infractores estudiantes de $4 .^{\circ}$ de la ESO, con las mismas edades y proporción en base al género que la muestra de jóvenes infractores. Para ello, a partir de una población de aproximadamente veinticinco mil estudiantes, mediante un muestreo aleatorio, multietápico, estratificado por provincia, tipo de centro, género y edad, se seleccionó una muestra de 399 participantes $(\alpha=0,5, z=1,96)$.

Tanto los responsables de los centros como los adolescentes fueron informados de la finalidad del estudio, estos últimos fueron invitados a participar de forma anónima y voluntaria, previa autorización de padres y/o tutores, garantizándoles la confidencialidad de la información y la protección de los datos. La tasa de rechazo se situó en el $1 \%$ de la población.

\section{Diseño}

El diseño de esta investigación responde a un modelo descriptivo de corte transversal mediante encuestas con muestras probabilísticas.

\section{Análisis de datos}

Para el análisis de datos se recurrió a la utilización de técnicas de estadística descriptiva univariada y multivariada, utilizando estadísticos de tendencia central y de dispersión (medias y desviaciones típicas), análisis de frecuencias y porcentajes, contrastes analíticos mediante análisis de varianza (ANOVA) y chi cuadrado; finalmente se realizaron sendos análisis de regresión logística binaria, con el objeto de identificar los predictores confiables del consumo y del policonsumo de drogas. Los datos fueron analizados mediante el programa estadístico SPSS 19.0 para Windows. 
Las medidas utilizadas en este estudio fueron el consumo de sustancias adictivas (alguna vez y el último mes) y la frecuencia, así como la edad de inicio en el consumo de las mismas. Por otra parte, con la finalidad de responder al objetivo principal de este estudio, como variable de contraste, se ha utilizado el tipo de muestra (adolescentes en infractores versus no infractores). Si bien para profundizar en los análisis de regresión fueron introducidas como variables predictoras todos los factores sociodemográficos y toxicológicos.

\section{RESULTADOS}

La muestra de adolescentes escolarizados no infractores y la de adolescentes infractores fueron equivalentes en base a la edad, el género y el nivel de cualificación. De las variables sociodemográficas estudiadas, únicamente se detectaron diferencias estadísticamente significativas en relación a la variable nivel de instrucción de los padres $\left(\mathrm{X}^{2}=56,38, \mathrm{p}=, 000\right)$.

El análisis de la prevalencia global de consumo de sustancias en las dos submuestras (adolescentes no infractores versus en infractores), revelan diferencias estadísticamente significativas entre ambos grupos $\left(\mathrm{X}^{2}=10,87, \mathrm{p}<, 001\right)$; el porcentaje de adolescentes que consumen alguna sustancia es más elevado en el grupo de jóvenes infractores $(73,9 \%)$ que en los no infractores $(55,1 \%)$. Asimismo, en el grupo de consumidores, se han detectados diferencias significativas en el porcentaje de policonsumidores $\left(\mathrm{X}^{2}=35,48, \mathrm{p}<, 000\right)$ y en el número de sustancias consumidas $(\mathrm{F}=68,56$, $\mathrm{p}<, 000)$; así, el $85,3 \%$ de los adolescentes sometidos a medidas judiciales refirió consumir más de dos sustancias, frente al 44,1\% de los no infractores, siendo la media de sustancias consumidas de $2,81(\mathrm{Sx}=1,26)$ y 1,64 $(\mathrm{S} x=0,92)$, respectivamente.

Las sustancias más consumidas en los adolescentes de este estudio, como era de esperar, son el tabaco, el alcohol y el cannabis, obteniéndose prevalencias de consumo muy superiores al resto de otras drogas. Para los jóvenes no infractores la sustancia más consumida es el alcohol $(79,7 \%)$, seguida del tabaco $(43,4 \%)$ y del cannabis $(24,8 \%)$; en el colectivo de adolescentes infractores, el orden y la prevalencia de las mismas varía ligeramente: tabaco y alcohol con igual porcentaje $(69,6 \%)$ seguidos de cannabis $(59,8 \%)$. 
Tabla 1

Consumo de sustancias adictivas (alguna vez) de los/as menores en función de la muestra

\begin{tabular}{|c|c|c|c|c|c|c|c|}
\hline \multirow{2}{*}{\multicolumn{2}{|c|}{ Sustancias }} & \multirow{2}{*}{\multicolumn{2}{|c|}{$\begin{array}{c}\text { No infractores } \\
(n=399)\end{array}$}} & \multirow{2}{*}{\multicolumn{2}{|c|}{$\begin{array}{c}\text { Infractores } \\
(n=92)\end{array}$}} & \multirow{3}{*}{$\begin{array}{c}\text { Valor } \\
\mathbf{X}^{2} \\
\end{array}$} & \multirow{3}{*}{$\begin{array}{c}\text { Porcentaje } \\
\text { Sig. }\end{array}$} \\
\hline & & & & & & & \\
\hline & & $\mathbf{n}$ & $\%$ & $\mathbf{n}$ & $\%$ & & \\
\hline \multirow{2}{*}{ Tabaco } & Sí & 173 & 43,4 & 64 & 69,6 & \multirow{2}{*}{20,56} & \multirow{2}{*}{,000 } \\
\hline & $\mathrm{No}$ & 226 & 56,6 & 28 & 30,4 & & \\
\hline \multirow{2}{*}{ Alcohol } & Sí & 318 & 79,7 & 64 & 69,6 & \multirow{2}{*}{4,44} & \multirow{2}{*}{,035 } \\
\hline & No & 81 & 20,3 & 28 & 30,4 & & \\
\hline \multirow{2}{*}{ Cannabis } & Sí & 99 & 24,8 & 55 & 59,8 & \multirow{2}{*}{42,47} & \multirow{2}{*}{,000 } \\
\hline & $\mathrm{No}$ & 300 & 75,2 & 37 & 40,2 & & \\
\hline \multirow{2}{*}{ Heroína* } & Sí & 1 & 0,3 & 2 & 2,2 & \multirow{2}{*}{4,55} & \multirow{2}{*}{,033 } \\
\hline & No & 398 & 99,7 & 90 & 97,8 & & \\
\hline \multirow{2}{*}{ Cocaína* } & Sí & 9 & 2,3 & 26 & 28,3 & \multirow{2}{*}{76,37} & \multirow{2}{*}{, 000} \\
\hline & No & 390 & 97,7 & 66 & 71,7 & & \\
\hline \multirow{2}{*}{ Sintéticas* } & Sí & 5 & 1,3 & 10 & 10,9 & \multirow{2}{*}{23,34} & \multirow{2}{*}{,000 } \\
\hline & No & 394 & 98,7 & 82 & 89,1 & & \\
\hline \multirow{2}{*}{$\begin{array}{l}\text { Otras sus- } \\
\text { tancias* }\end{array}$} & Sí & 7 & 1,8 & 5 & 5,4 & \multirow{2}{*}{4,25} & \multirow{2}{*}{,039 } \\
\hline & No & 392 & 98,2 & 87 & 94,6 & & \\
\hline
\end{tabular}

Nota: Teniendo en cuenta el reducido tamaño muestral en esta sustancia, debemos ser cautelosos en su interpretación.

Por sustancias, las desigualdades en el consumo entre ambos grupos se manifiestan igualmente. Los datos obtenidos muestran que salvo en el caso del alcohol, el consumo reportado para las demás sustancias es bastante más alto para los adolescentes infractores (ver Tabla 1). Se detectaron diferencias estadísticamente significativas en el consumo de todas las sustancias adictivas: tabaco $\left(\mathrm{X}^{2}=20,56, \mathrm{p}<, 000\right)$, alcohol $\left(\mathrm{X}^{2}=4,44, \mathrm{p}<, 035\right)$, cannabis $\left(\mathrm{X}^{2}=42,47, \mathrm{p}<, 000\right)$, heroína $\left(\mathrm{X}^{2}=4,55, \mathrm{p}<, 033\right)$, cocaína $\left(\mathrm{X}^{2}=76,37, \mathrm{p}<, 000\right)$, drogas de síntesis $\left(\mathrm{X}^{2}=23,34, \mathrm{p}<, 000\right) \mathrm{y}$ «otras sustancias» $\left(\mathrm{X}^{2}=4,25, \mathrm{p}<, 039\right)$. Destacan en las prevalencias de consumo que casi el $60 \%$ de estos jóvenes infractores consuma cannabis - porcentaje 2,5 veces superior al resto de los adolescentes-, el 28,3\% consuma cocaína - 12 veces más que el grupo no infractores-y que el 10,9\% consuma drogas de síntesis (consumo 8 veces superior). Por último, en relación al alcohol indicar que la tendencia cambia el sentido y son los jóvenes no infractores los que presentan consumos más elevados, con una prevalencia de 10 puntos porcentuales superior. 
Cuando analizamos la prevalencia de consumo de sustancias, en la muestra de adolescentes no infractores, no se detectaron diferencias estadísticamente significativas entre chicos y chicas, en ninguna de las sustancias estudiadas. Por el contrario, en la muestra de adolescentes infractores, únicamente se detectaron diferencias estadísticamente significativas en la prevalencia de consumo de heroína $\left(X^{2}=4,23, p<, 040\right)$. No obstante, teniendo en cuenta el reducido número de consumidores de esta sustancia debemos ser cautelosos en su interpretación.

Tabla 2

Consumo de sustancias adictivas (en el último mes) de los/as menores en función de la muestra

\begin{tabular}{|c|c|c|c|c|c|c|c|}
\hline \multirow{2}{*}{\multicolumn{2}{|c|}{ Sustancias }} & \multirow{2}{*}{\multicolumn{2}{|c|}{$\frac{\text { No infractores }}{(n=399)}$}} & \multirow{2}{*}{\multicolumn{2}{|c|}{$\begin{array}{c}\text { Infractores } \\
(n=92)\end{array}$}} & \multirow{3}{*}{$\begin{array}{c}\text { Valor } \\
\mathbf{X}^{2} \\
\end{array}$} & \multirow{3}{*}{$\begin{array}{c}\text { Porcentaje } \\
\text { Sig. }\end{array}$} \\
\hline & & & & & & & \\
\hline & & $\mathbf{N}$ & $\%$ & $\mathbf{N}$ & $\%$ & & \\
\hline \multirow{2}{*}{ Tabaco } & Sí & 98 & 25,9 & 63 & 68,5 & \multirow{2}{*}{59,77} & \multirow{2}{*}{,000 } \\
\hline & No & 281 & 74,1 & 29 & 31,5 & & \\
\hline \multirow{2}{*}{ Alcohol } & Sí & 198 & 52,0 & 57 & 62,0 & \multirow{2}{*}{2,97} & \multirow{2}{*}{,085 } \\
\hline & No & 183 & 48,0 & 35 & 38,0 & & \\
\hline \multirow{2}{*}{ Cannabis } & Sí & 50 & 13,3 & 41 & 44,6 & \multirow{2}{*}{46,34} & \multirow{2}{*}{, 000} \\
\hline & No & 327 & 86,7 & 51 & 55,4 & & \\
\hline \multirow{2}{*}{ Cocaína* } & Sí & 6 & 1,6 & 19 & 20,7 & \multirow{2}{*}{53,24} & \multirow{2}{*}{,000 } \\
\hline & No & 371 & 98,4 & 73 & 79,3 & & \\
\hline \multirow{2}{*}{ Sintéticas* } & Sí & 5 & 1,3 & 7 & 7,6 & \multirow{2}{*}{11,71} & \multirow{2}{*}{,001 } \\
\hline & No & 372 & 98,7 & 85 & 92,4 & & \\
\hline \multirow{2}{*}{$\begin{array}{l}\text { Otras } \\
\text { sustancias* }\end{array}$} & Sí & 8 & 2,5 & 4 & 4,3 & \multirow{2}{*}{0,85} & \multirow{2}{*}{, 356} \\
\hline & $\mathrm{No}$ & 311 & 97,5 & 88 & 95,7 & & \\
\hline
\end{tabular}

Nota: Teniendo en cuenta el reducido tamaño muestral en esta sustancia, debemos ser cautelosos en su interpretación.

Un patrón similar, con ligeras matizaciones, puede detectarse en el análisis del consumo en el último mes (ver Tabla 2). Para todas las sustancias, sin excepción, se detecta mayor representación porcentual de consumo en los jóvenes infractores, llegando las diferencias a tener significación estadística para las siguientes: tabaco $\left(\mathrm{X}^{2}=59,77, \mathrm{p}<, 000\right)$, cannabis $\left(\mathrm{X}^{2}=46,34\right.$, $\mathrm{p}<, 000)$, cocaína $\left(\mathrm{X}^{2}=53,24, \mathrm{p}<, 000\right)$ y drogas de síntesis $\left(\mathrm{X}^{2}=11,71, \mathrm{p}<, 001\right)$.

El análisis de la prevalencia de consumo en el último mes en la muestra de adolescentes no infractores en función del género reveló la existencia de diferencias estadísticamente significativas en el consumo de tabaco $\left(X^{2}=4,41\right.$, $\mathrm{p}<, 0,25)$ y alcohol $\left(\mathrm{X}^{2}=4,84, \mathrm{p}<, 018\right)$, siendo las chicas las que presentan por- 
centajes de consumo más elevados. Por el contrario, no se detectaron diferencias estadísticamente significativas entre los chicos y chicas infractores.

Tabla 3

Frecuencia y porcentajes de consumo en el último mes para la población de consumidores de sustancias

\begin{tabular}{|c|c|c|c|c|c|c|c|}
\hline \multicolumn{2}{|c|}{ Sustancias } & \multicolumn{2}{|c|}{ No infractores } & \multicolumn{2}{|c|}{ Infractores } & \multirow{2}{*}{$\begin{array}{c}\text { Valor } \\
\mathbf{X}^{2}\end{array}$} & \multirow{2}{*}{$\begin{array}{c}\begin{array}{c}\text { Porcen } \\
\text { taje }\end{array} \\
\text { Sig. } \\
\end{array}$} \\
\hline & & $\mathbf{n}$ & $\%$ & $\mathbf{n}$ & $\%$ & & \\
\hline \multirow{3}{*}{ Tabaco } & Diariamente & 43 & 43,9 & 59 & 93,7 & \multirow{3}{*}{41,54} & \multirow{3}{*}{,000 } \\
\hline & $\begin{array}{l}1 \text { o más veces } \\
\text { semana }\end{array}$ & 20 & 20,4 & 3 & 4,8 & & \\
\hline & $\begin{array}{l}\text { Menos } 1 \text { vez } \\
\text { semana }\end{array}$ & 35 & 35,7 & 1 & 1,6 & & \\
\hline \multirow{3}{*}{ Alcohol } & Diariamente & 7 & 3,5 & 7 & 12,3 & \multirow{3}{*}{25,13} & \multirow{3}{*}{,000 } \\
\hline & $\begin{array}{l}1 \text { o más veces } \\
\text { semana }\end{array}$ & 107 & 54,0 & 45 & 78,9 & & \\
\hline & $\begin{array}{l}\text { Menos } 1 \text { vez } \\
\text { semana }\end{array}$ & 84 & 42,5 & 5 & 8,8 & & \\
\hline \multirow{3}{*}{ Cannabis } & Diariamente & 5 & 10,0 & 25 & 61,0 & \multirow{3}{*}{28,29} & \multirow{3}{*}{, 000} \\
\hline & $\begin{array}{l}1 \text { o más veces } \\
\text { semana }\end{array}$ & 21 & 42,0 & 11 & 26,8 & & \\
\hline & $\begin{array}{l}\text { Menos } 1 \text { vez } \\
\text { semana }\end{array}$ & 24 & 48,0 & 5 & 12,2 & & \\
\hline \multirow{3}{*}{ Cocaína* } & Diariamente & 1 & 16,7 & 2 & 10,5 & \multirow{3}{*}{2,44} & \multirow{3}{*}{,295 } \\
\hline & $\begin{array}{l}1 \text { o más veces } \\
\text { semana }\end{array}$ & 2 & 33,3 & 13 & 68,4 & & \\
\hline & $\begin{array}{l}\text { Menos } 1 \mathrm{vez} \\
\text { semana }\end{array}$ & 3 & 50,0 & 4 & 21,1 & & \\
\hline \multirow{3}{*}{ Sintéticas* } & Diariamente & 2 & 40,0 & 0 & 0,0 & \multirow{3}{*}{3,77} & \multirow{3}{*}{, 152} \\
\hline & $\begin{array}{l}1 \text { o más veces } \\
\text { semana }\end{array}$ & 2 & 40,0 & 6 & 85,7 & & \\
\hline & $\begin{array}{l}\text { Menos } 1 \text { vez } \\
\text { semana }\end{array}$ & 1 & 20,0 & 1 & 14,3 & & \\
\hline
\end{tabular}




\begin{tabular}{|c|c|c|c|c|c|c|c|}
\hline \multicolumn{2}{|c|}{ Sustancias } & \multicolumn{2}{|c|}{ No infractores } & \multicolumn{2}{|c|}{ Infractores } & \multirow{2}{*}{$\begin{array}{c}\text { Valor } \\
\mathbf{X}^{2}\end{array}$} & \multirow{2}{*}{$\begin{array}{c}\begin{array}{c}\text { Porcen- } \\
\text { taje }\end{array} \\
\text { Sig. }\end{array}$} \\
\hline & & $\mathbf{n}$ & $\%$ & $\mathbf{n}$ & $\%$ & & \\
\hline \multirow{3}{*}{$\begin{array}{l}* \text { Otras } \\
\text { sustancias }\end{array}$} & Diariamente & 4 & 50,0 & 1 & 25,0 & \multirow{3}{*}{0,90} & \multirow{3}{*}{,638 } \\
\hline & $\begin{array}{l}1 \text { o más veces } \\
\text { semana }\end{array}$ & 2 & 25,0 & 1 & 25,0 & & \\
\hline & $\begin{array}{l}\text { Menos } 1 \text { vez } \\
\text { semana }\end{array}$ & 2 & 25,0 & 2 & 50,0 & & \\
\hline
\end{tabular}

Nota: Teniendo en cuenta el reducido tamaño muestral en esta sustancia, debemos ser cautelosos en su interpretación.

Finalmente, en la frecuencia de consumo en el último mes -entre la población consumidora de las diferentes sustancias- (ver Tabla 3) se han constatado diferencias significativas en tabaco $\left(\mathrm{X}^{2}=41,54, \mathrm{p}<, 000\right)$, alcohol $\left(\mathrm{X}^{2}=25,13, \mathrm{p}<, 000\right)$ y cannabis $\left(\mathrm{X}^{2}=28,29, \mathrm{p}<, 000\right)$. El consumo habitual está más presente en los y las adolescentes infractores: un 93,7\% de estos consumen diariamente tabaco, un 12,3\% alcohol y un $61 \%$ cannabis; en los adolescentes no infractores se percibe un consumo más esporádico, ya que los porcentajes de uso diario descienden a $43,9 \%, 3,5 \%$ y $10 \%$, respectivamente.

El análisis de las frecuencias de consumo en función del género reveló que los chicos no infractores presentan consumos más frecuentes que los de las chicas $\left(X^{2}=9,40, p<, 011\right)$ en relación al alcohol, no detectándose diferencias en relación a las demás sustancias. Por su parte, los adolescentes infractores no presentaron diferencias en función del género en relación a las frecuencias de consumo de las sustancias estudiadas.

Tabla 4

Edades de inicio en el consumo de sustancias adictivas en función de la muestra

\begin{tabular}{|c|c|c|c|c|c|c|c|c|}
\hline \multirow{2}{*}{ Sustancias } & \multicolumn{3}{|c|}{ No infractores } & \multicolumn{3}{|c|}{ Infractores } & \multirow{2}{*}{$\begin{array}{c}\text { Valor } \\
\text { F } \\
\end{array}$} & \multirow{2}{*}{$\begin{array}{c}\text { Porcentaje } \\
\text { Sig. } \\
\end{array}$} \\
\hline & $\mathbf{n}$ & Media & $\left(\mathbf{S}_{\mathrm{x}}\right)$ & $\mathbf{n}$ & Media & $\left(\mathbf{S}_{\mathrm{x}}\right)$ & & \\
\hline Tabaco & 139 & 13,12 & 2,14 & 63 & 11,96 & 1,95 & 13,44 &, 000 \\
\hline Alcohol & 208 & 13,44 & 1,70 & 62 & 13,29 & 1,56 & 0,39 &, 530 \\
\hline Cannabis & 89 & 14,33 & 1,21 & 53 & 13,30 & 1,39 & 21,56 & ,000 \\
\hline Heroína* & 1 & 15 & - & 2 & 14 & 1,41 & 0,33 & 667 \\
\hline Cocaína* & 9 & 14,55 & 1,51 & 26 & 14,69 & 0,79 & 1,22 & ,729 \\
\hline Sintéticas* & 5 & 15,60 & 1,14 & 10 & 14,70 & 0,82 & 3,11 & 101 \\
\hline
\end{tabular}




\begin{tabular}{lccccccccc}
\hline \multirow{2}{*}{ Sustancias } & \multicolumn{3}{c}{ No infractores } & \multicolumn{3}{c}{ Infractores } & \multicolumn{2}{c}{ Valor } & Porcentaje \\
\cline { 2 - 9 } & $\mathbf{n}$ & Media & $\left(\mathbf{S}_{\mathbf{x}}\right)$ & $\mathbf{n}$ & Media & $\left(\mathbf{S}_{\mathbf{x}}\right)$ & $\mathbf{F}$ & Sig. \\
\hline $\begin{array}{l}\text { Otras sus- } \\
\text { tancias* }\end{array}$ & 7 & 14,42 & 1,61 & 5 & 14,60 & 0,89 & 0,45 &, 836 \\
\hline
\end{tabular}

Nota: Teniendo en cuenta el reducido tamaño muestral en esta sustancia, debemos ser cautelosos en su interpretación.

En relación a la edad de inicio de los consumos (ver Tabla 4), se detectaron diferencias estadísticamente significativas entre ambos grupos con respecto al tabaco $(\mathrm{F}=13,44, \mathrm{p}<, 000)$ y cannabis $(\mathrm{F}=21,56, \mathrm{p}<, 000)$, siendo más precoces en utilizar estas sustancias los chicos y chicas infractores; las edades medias de inicio para este colectivo se sitúan en $11,96(\mathrm{Sx}=1,95)$ y $13,30(\mathrm{~S} x=1,39)$ respectivamente, y para los jóvenes no infractores en 13,12 $(\mathrm{S} x=2,14)$ y $14.33(\mathrm{Sx}=1,21)$. Además, sin que las diferencias sean significativas, se observa una tendencia similar en el inicio de consumo de alcohol, heroína y drogas de síntesis.

Si segmentamos los análisis en función del tipo de muestra y del género, observamos que en el grupo de adolescentes no infractores existen diferencias en las edades de inicio en el consumo de sustancias entre chicos y chicas en relación al tabaco y alcohol, siendo los primeros quienes refieren tener un inicio más precoz en el consumo de ambas sustancias. Por su parte, no se detectaron diferencias estadísticamente significativas entre los chicos y las chicas infractores.

Por último, en la figura 1, se presentan los resultados encontrados en los modelos finales de los análisis de regresión logística binaria por pasos hacia adelante, indicando el coeficiente de determinación (porcentaje de varianza explicada) y la estimación de los coeficientes con su respectiva prueba estadística y sus intervalos de confianza del 95\% para intentar predecir el consumo y el policonsumo de sustancias. Para el modelo consumo de drogas se utilizaron como variables independientes las variables sociodemográficas (edad, género, historial académico, rendimiento académico y estudios de los padres). Mientras que para el modelo policonsumo las variables independientes fueron el historial toxicológico y las variables sociodemográficas.

Consumo de Sustancias: Para los adolescentes no infractores el modelo consumo de drogas presenta un coeficiente de determinación $\mathrm{R}^{2} \mathrm{c}=, 444$; que únicamente es explicado positivamente por la variable Edad. Mientras que para los adolescentes infractores el modelo consumo de drogas presenta un coeficiente de determinación $\mathrm{R}^{2} \mathrm{c}=, 498$; que únicamente es explicado negativamente por la variable estudios de los padres. 


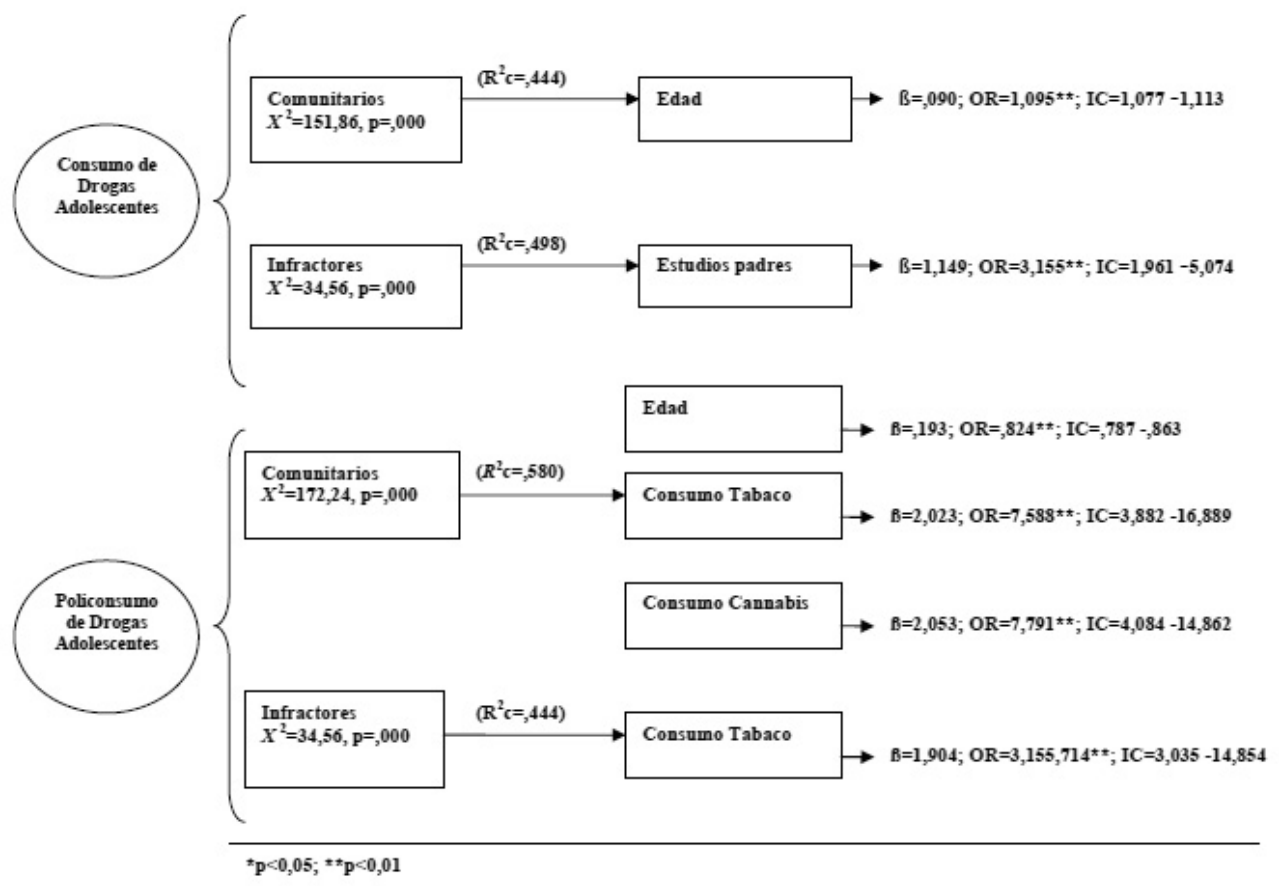

Figura 1. Modelos de regresión logística binaria para consumo y policonsumo de sustancias en adolescentes no infractores e infractores

Policonsumo de Sustancias: Para los adolescentes no infractores el modelo policonsumo de drogas presenta un coeficiente de determinación $\mathrm{R}^{2} \mathrm{c}=, 580$; que es explicado positivamente por las variables consumo de tabaco y consumo de cannabis y negativamente por la edad. Mientras que para los adolescentes infractores el modelo policonsumo de drogas presenta un coeficiente de determinación $\mathrm{R}^{2} \mathrm{c}=, 444$; que únicamente es explicado positivamente por la variable consumo de tabaco.

\section{DISCUSIÓN}

Los resultados de este estudio reflejan que las sustancias más consumidas entre los adolescentes gallegos escolarizados en $4 .{ }^{\circ}$ de la ESO, globalmente considerados, son fundamentalmente el alcohol, el tabaco y en tercer lugar, el cannabis, obteniéndose prevalencias de consumo muy superiores al resto de sustancias (drogas de síntesis, heroína, cocaína...). Esta tendencia es consistente con lo reportado en diferentes estudios europeos (Björn, et al., 2012), nacionales (DGPNSD, 2011) y autonómicos (Giménez, 2009; Xunta de Galicia, 2011). Estos datos nos plantean la necesidad de advertir 
lo que ha sido indicado consistentemente en relación a la progresión en el consumo de drogas legales a las ilegales (Becoña, 2002); concordando con la denominada teoría de la escalada (Kandel y Davies, 1992). Si bien esta secuencia solo está presente en un porcentaje determinado de sujetos (Soria, 2006), ya que no debemos olvidar que el consumo de una droga específica está influenciada no solo por el consumo de drogas previas, sino por otros factores como la personalidad, el estilo de vida y los factores ambientales, como señalan Yamaguchi y Kandel (1985).

Sin embargo, el aspecto más relevante de este estudio es que aporta datos sobre las prevalencias de consumo en jóvenes infractores, aspecto que ha sido poco documentado en nuestro país. Estos adolescentes, en comparación con sus homólogos no infractores, presentan unos porcentajes de consumo de sustancias significativamente más elevados (DGPNSD, 2011; Gamonal, Insua y Moreno, 2009; Observatorio Europeo de las Drogas y las Toxicomanías, 2003). En nuestro estudio, el 73,9\% de estos jóvenes refirió haber consumido alguna sustancia adictiva, frente al $55,1 \%$ de los no infractores; detectándose asimismo un porcentaje de policonsumidores superior en el mismo grupo; diferencias entre ambos que alcanzaron significación estadística. Cabe mencionar, no obstante, que no toda la población infractora está involucrada en el consumo de drogas. Es decir, no todos los menores que viven en situaciones sociales deficitarias incurren en el consumo de sustancias adictivas (Comas, Aguinaga, Andrés et al., 2003; Pantoja y Añañós, 2010).

El análisis del consumo por sustancias es más elevado en los menores infractores en comparación con la población no infractora de sus mismas edades, con la única excepción del alcohol. Las sustancias adictivas ilegales más consumidas en el colectivo de adolescentes infractores son el cannabis, la cocaína y las drogas de síntesis al igual que sucede con muestras no infractoras (Lomba, Apóstolo y Mendes, 2009; Merino, 2008; Ministerio de Sanidad, Servicios Sociales e Igualdad, 2011). No obstante, se manifiestan desigualdades significativas entre ambos colectivos en las prevalencias. El consumo más elevado, en el grupo de jóvenes infractores, en cocaína, drogas de síntesis y, sobre todo, en cannabis, coincide con la tendencia marcada en otros estudios de poblaciones con similares características (Esteban, et al., 2003; Gamonal, Insua y Moreno, 2009; ICBF/DNE, 2010).

Cuando analizamos el consumo de sustancias en función del género, segmentado en función de las muestras de adolescentes estudiados (no infractores versus infractores), en general observamos una prevalencia de consumo similar entre chicos y chicas, únicamente se detectaron diferencias estadísticamente significativas en el grupo de adolescentes infractores en relación al consumo de heroína, siendo las chicas las que refieren mayor 
consumo de esta sustancia. Estos datos apuntan un posible cambio en las pautas de consumo de chicos y chicas (Mendoza, López y Sagrera, 2007). Una tendencia similar se observa cuando analizamos las prevalencias de consumo el último mes, pues únicamente se detectaron diferencias estadísticamente significativas en función del género en el grupo de adolescentes no infractores en relación al consumo de alcohol y tabaco, siendo además estos consumos más prevalentes en el grupo de chicas. Estos datos van en la línea de lo reportado en estudios recientes de ámbito nacional (DGPNSD, 2011) y ponen de manifiesto que se está produciendo una igualación e incluso inversión en los consumos de sustancias que tradicionalmente eran más prevalentes en varones (Mendoza, López y Sagrera, 2007).

Si profundizamos en los análisis observamos que en el grupo de adolescentes no infractores a pesar de que las chicas tienen consumos similares, o incluso superiores en el caso de alcohol y tabaco, este consumo es realizado menos frecuentemente que sus homólogos varones. Sin embargo, cuando analizamos las frecuencias de consumo en función del género en la muestra de adolescentes infractores no se detectaron diferencias estadísticamente significativas para ninguna de las sustancias estudiadas, lo que indica que en este colectivo los patrones de consumo de sustancias son muy similares.

Por tanto, nuestros datos vienen a corroborar lo que, en los últimos años, la literatura sobre prevención de drogas viene reportando: la importante reducción de las diferencias inter-género. En este sentido los datos epidemiológicos señalan una tendencia a la igualación entre géneros, e incluso inversión de los patrones de consumo en drogas institucionalizadas, radicando el problema en que estas diferencias no han disminuido debido a un descenso drástico en los grupos de consumidores masculinos, sino por un aumento importante de consumo por parte de las chicas (DGPNSD, 2011; Mendoza, López y Sagrera, 2007; Ministerio de Sanidad y Política Social, 2009).

El perfil de los adolescentes infractores presenta una gran precocidad en el inicio del consumo de drogas situándolos en un nivel de riesgo mayor que los adolescentes no infractores (Contreras, Molina y Cano, 2012; DíazAguado, Martínez y Martín, 2012; Esteban et al, 2003; Gamonal, Insua y Moreno, 2009; ICBF y DNE, 2010; San Juan, Ocariz y German, 2009). Cuando analizamos las edades de inicio en los consumos de sustancias, observamos que los adolescentes infractores se inician más precozmente. En este sentido, existe evidencia de que los adolescentes que consumen drogas a edades muy tempranas tienen más problemas de indisciplina, rendimiento o de fracaso escolar (Casas, 1998). Asimismo, tal como señalan Graña, Garrido y González (2007), el consumo prematuro de sustancias adictivas está direc- 
tamente relacionado con las actividades predelictivas, y además actúa como una variable predictora de la reincidencia delictiva. Esta relación no es de causalidad, ni tampoco se conoce cuál es el orden de los factores droga-delincuencia versus delincuencia-droga (UNODC, 2010). En esta línea, Barnes y Welte (1986) refirieron que a más abuso de drogas, más probabilidades hay de que persista la conducta antisocial.

Por otra parte, el consumo de drogas tiene una repercusión negativa en la conducta de los adolescentes a nivel psicológico, psicopatológico, escolar y social. En este sentido, estudios realizados en el contexto nacional e internacional reflejan que los adolescentes que consumen alcohol y otras drogas presentan mayor incidencia del trastorno negativista desafiante, trastornos disociales y otros cuadros clínicos como la depresión o la hiperactividad (Casas, 1998; Pettersen y Skrondal, 1999). Asimismo, algunos estudios indican que el abuso de sustancias puede estar relacionado con mayores tasas de infecciones de transmisión sexual y de embarazos no deseados (Ellickson, McCaffrey y Klein, 2009).

En relación a los análisis de regresión logística practicados para predecir el consumo de sustancias, en la muestra de adolescentes no infractores se identificó la variable edad como un predictor confiable del consumo de drogas; estos datos van en la línea de lo esperado, pues como sabemos, en el intervalo de edad de los participantes (14-17), el consumo de las sustancias estudiadas suele incrementarse progresivamente, hasta alcanzar su punto máximo en el intervalo de los 18 a los 24 años (DGPNSD, 2011). Por lo que se refiere a la muestra de jóvenes infractores, la variable predictora del consumo de sustancias fue el estudio de los padres, en concreto el bajo nivel de formación de los padres se asoció al consumo; tendencia que va en la línea de lo informado en otros estudios en los que se señala que el bajo nivel de instrucción de los padres es un factor de riesgo para el consumo (Villena, et al., 2009). Llama la atención que, en estos mismos análisis de regresión, otras variables sociodemográficas relevantes, por ejemplo, el historial o el rendimiento académico, no se hayan identificado como predictores del consumo de drogas tal como ha sido reportado en otros estudios (López y Rodríguez-Arias, 2010; González y Laespada, 2012); aspecto que tendremos en cuenta en futuras investigaciones. Pudiéramos aventurar que la razón subyace a la homogeneidad de las características intragrupos, ya que se trata de infractores que todavía no han abandonado la escuela y, como sabemos, la deserción escolar está muy relacionada en el abuso de drogas.

Asimismo, por lo que se refiere a los análisis de regresión logística para policonsumo de drogas, en la muestra de adolescentes no infractores, el consumo previo de tabaco y cannabis fueron identificados como predictores 
confiables, estos datos son consistentes con lo reportado por la teoría de la escalada (Kandel y Davies, 1992). Sin embargo, la edad explicó el modelo de forma negativa; una posible explicación a este dato se deriva del hecho de que la muestra está integrada por adolescentes que se mantienen en el sistema educativo, lo que tal vez pueda convertirse en un factor de protección por cuanto que es un indicador de integración escolar (Navarro, 1992). No obstante, este aspecto debiera ser objeto de mayor atención en futuros estudios. Por lo que se refiere a la muestra de adolescentes infractores, el consumo de tabaco actuó como un predictor del policonsumo de sustancias, resultado que va en la línea de lo esperado dado que el tabaco es una de las sustancias que más habitualmente se utiliza en combinación con otras sustancias (Viña y Herrero, 2004).

Las conclusiones derivadas de este estudio sugieren, por una parte, incrementar la investigación acerca de los menores/adolescentes infractores respecto al abuso de drogas y, por otra parte, abordar de forma sistemática la prevención y el tratamiento del consumo de sustancias, tal como se contempla en la Ley Orgánica 5/2000 de Responsabilidad Penal del Menor. Debemos tener en cuenta que un porcentaje significativo de estos jóvenes abandonan temprano el sistema educativo, quedándose fuera de las actuaciones de prevención universal, por lo que es necesario desarrollar e implementar también proyectos de prevención selectiva e indicada. En consecuencia, estos programas debieran abordar la prevención de las drogodependencias desde un enfoque global entrenando a estos adolescentes, no solo en habilidades específicas para rechazar el ofrecimiento de sustancias, sino también en habilidades generales para la vida que les permitan afrontar eficazmente situaciones adversas o de riesgo para el consumo $(\mathrm{Gu}-$ xens et al., 2007; Pantoja y Añaños, 2010). Las propuestas actuales más eficaces hacen, por tanto, hincapié en el diseño e implementación de programas multicomponente; no se trata solamente de incrementar conocimientos sobre esta temática, sino de entrenar a los adolescentes en habilidades generales que les "preparen para la vida», en definitiva, para una toma de decisiones responsable. La finalidad de estos programas es prevenir que el uso y abuso de drogas desencadene una trayectoria delictiva o la agrave, así como proteger su salud y desarrollo integral. En esta línea, ya se han realizado algunas iniciativas en nuestro país. Según datos del PNSD (2011), un total de 371 menores infractores han sido atendidos en programas terapéuticos y de prevención en centros de menores de Castilla y León, Cataluña, Ceuta, Madrid..., pero todavía falta mucho por hacer. Puede ser de ayuda la obra coordinada por Pantoja y Añaños (2010) en la que se recogen ejemplos de intervenciones educativas que, centradas en la prevención selectiva, se han desarrollado en distintos países con menores vulnerables. Asimismo, los resultados de estas investigaciones debieran ser útiles para plantear tratamientos rehabilitadores especializados y focaliza- 
dos en este colectivo, hasta la fecha escasos (UNODC, 2010), rompiendo las barreras existentes en el sistema actual y poniendo a disposición de estos adolescentes programas de prevención y de tratamiento basados en la evidencia científica.

Finalmente, consideramos necesario hacer alusión a las limitaciones de este estudio. Si bien hemos encuestado a la totalidad de adolescentes infractores escolarizados en cuarto de la ESO, la muestra es reducida y está limitada a una comunidad autónoma; por lo que en futuros estudios se requieren muestras más amplias y representativas del territorio nacional. Por otra parte, la presente investigación tiene un enfoque fundamentalmente descriptivo, aunque pudiera ser justificado por la escasez de investigaciones. Reconocemos, no obstante, la necesidad de contemplar en su análisis más variables biopsicosociales y educativas relacionadas con el consumo de sustancias que nos permitan identificar factores de riesgo y protección específicos de este colectivo, así como las interrelaciones consumo y actividad delictiva. 


\section{REFERENCIAS BIBLIOGRÁFICAS}

Andrade, R. C., Silva, V. A., \& Assumpção F. B. (2004). Preliminary data on the prevalence of psychiatric disorders in Brazilian male and female juvenile delinquents. Brazilian Journal of Medical and Biological Research, 37(8), 1155-1160.

Añaños, F., y Pantoja, L. (2010). Actuaciones socioeducativas con menores vulnerables, en riesgo, relacionados con la droga. Reflexiones críticas. Revista Interuniversitaria de Pedagogía Social, 17, 109-122.

Barnes, G., \& Welte, J. (1986). Patterns and Predictors of Alcohol Use among 7-12th Grade Students in New York State. Journal of Study on Alcohol, 47(1), 53-62.

Becoña, E. (2002). Bases científicas de la prevención de las drogodependencias. Madrid: Delegación del Gobierno para el Plan Nacional sobre Drogas.

Björn, H., Guttormsson, U., Ahlström, Balakireva, O., Bjarnson, T., Kokkevi, A., \& Graus, L. (2012). The 2011 ESPAD report substance use among students in 36 European countries. Stockholm: The Swedish Council for Information on Alcohol and Other Drugs (CAN). Recuperado de: http://alcoholireland.ie/download/ reports/alcohol_health/children_young_ people/the-2011-espad_report.pdf

Cabrera, J., y Filgueira, E. (dir) (2011). Xuventude Galega 2010. Santiago: Consellería de Traballo e Benestar.

Casas, J. (1998). Actitudes, habilidades sociales y autocontrol en la prevención del consumo abusivo de alcohol y tabaco. [Tesis doctoral]. Madrid: Universidad Autónoma de Madrid.

Comas, D., Aguinaga, J., Orizo, F. A., Espinosa, A., y Ochaita, E. (2003). Jóve- nes $y$ estilos de vida. Valores y riesgos de los jóvenes urbanos. Madrid: FAD e INJUVE.

CONACE (2002). Estudio acerca de la magnitud del consumo y factores asociados al uso de droga en la población de SEMANE. Santiago de Chile: Consejo Nacional para el Control de Estupefacientes (CONACE) y Servicio Nacional de Menores (SEMANE).

Contreras, L., Molina, V. y Cano, M. C. (2012). Consumo de drogas en adolescentes con conductas infractoras: análisis de variables psicosociales implicadas. Adicciones, 24(1), 31-38.

DGPNSD (2011). Encuesta estatal sobre uso de drogas en estudiantes de enseñanzas secundarias (ESTUDES), 19942010. Madrid: Ministerio de Sanidad y Política Social.

Díaz-Aguado, M. J., Martínez, M., y Martín, S. (2002). Génesis y desarrollo de los comportamientos de los jóvenes con problemas de conducta en centros de menores. Madrid: Ministerio de Trabajo y Asuntos Sociales.

Ellickson, P. L., McCaffrey, D. F., \& Klein, D. J. (2009). Long-term Effects of Drug Prevention on Risky Sexual Behavior among Young Adults. Journal of Adolescent Health, 45(2), 111-117.

Espada, J., Méndez, X., Griffin, K., y Botvin, G. (2003). Adolescencia: Consumo de alcohol y otras drogas. Revista papeles del psicólogo, 23(84), 9-17.

Esteban, B., Díaz, A, Gañan, A., García, J., Gordón, B., y Varela, C. (2003). Menores infractores con consumo de drogas en los centros de reforma: 2002/2003. Madrid: Servicio Interdisciplinar de Atención a las Drogodependencias. Recuperado de: http:// 
www.asociacionsiad.com/Documents/ Estudio2002.pdf

Gamonal, A., Insua, P., y Moreno, G. (2009). Proyecto de prevención y abordaje del consumo de drogas en centros de protección y de reeducación de Galicia. Fase I: Análisis de situación y evaluación de necesidades. Santiago de Compostela: Xunta de Galicia.

Giménez, F. J. (dir) (2009). Consumo de drogas en Galicia año 2008. Madrid: Edis. Recuperado de: http://www.sergas.es/cas/DocumentacionTecnica/ docs/Drogodependencias/Consumo_ drogas_Galicia_VolI.pdf

Gobierno Nacional de la República de Colombia (2009). Estudio de consumo de sustancias psicoactivas en adolescentes infractores de la ley en Colombia - 2009. Documento de Proyecto. Bogotá D. C: Gobierno Nacional de la República de Colombia.

González, J. M., y Laespada, M. T. (2012). La prevención selectiva en el ámbito educativo y las buenas prácticas. En M. Isorna y D. Saavedra (Coords). Prevención de drogodependencias y otras conductas adictivas (pp. 319-369). Madrid: Pirámide.

Graña, J. L., Garrido, V., y González, L. (2007). Reincidencia delictiva en menores infractores de la Comunidad de Madrid: Evaluación, caracteristicas delictivas y modelos de predicción. Madrid: Agencia de la Comunidad de Madrid para la Reeducación y Reinserción del Menor Infractor.

Guxens, M., Nebot, M., Ariza, C. y Ochoa, D. (2007). Factores asociados al inicio del consumo de cannabis: una revisión sistemática de estudios de cohortes. Gaceta Sanitaria, 19(3), 1-9.

Instituto Colombiano de Bienestar Familiar (ICBF) y la Dirección Nacional de Estupefacientes (DNE) (2010).
Estudio Nacional de consumo de sustancias psicoactivas en adolescentes en conflicto con la ley en Colombia 2009. Bogotá: Gobierno Nacional de la República de Colombia. Recuperado de: http://www.descentralizadrogas.gov. co/Portals/0/Estudio $\% 20$ Consumo $\% 20$ adolescentes.pdf

Instituto de Sociología (2007). Estudio de prevalencia y factores asociados en adolescentes infractores de ley. Santiago de Chile: Pontificia Universidad Católica de Chile, Facultad de Ciencias Sociales.

Kandel, D. B. \& Davies, M. (1992). Progression to regular marijuana involvement: Phenomenology and risk factors near-daily use. En M. Glantz, y R. Pickens (eds). Vulnerability to drug abuse. (pp. 211-253). Washington: American Psychological Association.

Ley Orgánica 5/2000, de 12 de enero, reguladora de la responsabilidad penal de los menores (BOE n. ${ }^{\circ} 11$ de 13 de enero de 2000).

Ley 3/2011 de 30 de junio, de 30 de junio, de apoyo a la familia y a la convivencia de Galicia. (BOE n. ${ }^{\circ} 182$ de 30 de julio de 2012).

Lindsay, G. \& Rainey, J. (1997). Psychosocial and pharmacological explanations of nicotine's "gateway drug» function. The Journal of School Health. 67(4), 123-126.

Lomba, L., Apóstolo, J., y Mendes, F. (2009). Consumo de drogas, alcohol y conductas sexuales en los ambientes recreativos nocturnos de Portugal. Adicciones, 21(4), 309-326.

López, S., y Rodríguez-Arias, J. L. (2010). Factores de riesgo y de protección en el consumo de drogas en adolescentes y diferencias según edad y sexo. Psicothema, 22(4), 568-573. 
Mendoza, R., López, P., y Sagrera, M. R. (2007). Diferencias de género en la evolución del tabaquismo adolescente en España (1986-2002). Adicciones, 19(3), 273-288.

Merino, B. (coord.) (2008). Ganar salud con la juventud. Nuevas recomendaciones sobre salud sexual y reproductiva, consumo de alcohol y salud mental. Madrid: Ministerio de Sanidad y Consumo e INJUVE.

Ministerio de Sanidad y Política Social (2009). Estrategia nacional sobre drogas 2009-2016. Madrid: Ministerio de Sanidad y Política Social.

Ministerio de Sanidad, Servicios Sociales e Igualdad (2011). Plan Nacional sobre Drogas. Memoria 2010. Madrid: Ministerio de Sanidad, Servicios Sociales e Igualdad.

Moreno, A. (1997). La adolescencia como tiempo de cambios. En J. A. García Madrugada, y P. Pardo de León (eds). Psicología Evolutiva. (pp. 258283). Madrid: Universidad Nacional de Educación a Distancia.

Navarro, J. (2002). Factores de riesgo y protección de carácter social relacionados con el consumo de drogas. Madrid: Ayuntamiento de Madrid.

Observatorio Europeo de las Drogas y las Toxicomanías (2003). Drogas en el punto de mira: El consumo de drogas entre la población joven más vulnerable. Septiembre-octubre. Luxemburgo: Oficina de Publicaciones Oficiales de las Comunidades Europeas.

Pedersen, W. \& Skrondal, A. (1999). Ecstasy and new patterns of drug use: anormal population study. Addiction, 94(11), $1695-1706$.
Sáenz, M. A., Bejarano J., y Fonseca, S. (2006). En condiciones de vulnerabilidad: Consumo de drogas en adolescentes privados de la libertad y transgresión de la ley penal. Revista de la Asociación de Ciencias Penales de Costa Rica. 18(24), 79-88.

San Juan, C., Ocáriz, E., y Germán, I. (2009). Menores infractores y consumo de drogas: perfil psicosocial y delictivo. Revista Criminalidad, 51(1), 147-162.

Soria, G. (2006). Sistemas cannabinoide y purinérgico: posibles sustratos neurobiológicos de la drogadicción [Tesis doctoral]. Barcelona: Universitat Pompeu Fabra. Recuperado de: http://www. tdx.cat/bitstream/handle/10803/7101/ tgsr.pdf? sequence $=1$

UNODC (2010) La relación droga y delito en adolescentes infractores de la ley la experiencia de Bolivia, Chile, Colombia, Perú y Uruguay. New York: Comisión Interamericana para el control del abuso de drogas.

Villena, A., Morena, S., Párraga, I., González, M. D., Soriano, H., y López-Torres, J. (2009). Factores asociados al consumo de Tabaco en Adolescentes. Revista Clínica de Medicina de Familia, 2(7), 320-325

Viña, C. M., y Herrero, M. (2004). El consumo de sustancias psicoactivas en estudiantes de Psicología de la Universidad de la Laguna. International Journal of Clinical and Health Psychology, 4(3), 521-536.

Yamaguchi, K. \& Kandel, D. (1985). On the resolution of role incompatibility: A life event history of analysis of family roles and marijuana use. American journal of Sociology, 90(6), 1284-1325. 


\section{PERFIL ACADÉMICO Y PROFESIONAL DE LOS AUTORES}

José María Faílde Garrido, Titular de Universidad en el Departamento de Análisis e Intervención Psicopedagogía, Miembro del grupo de investigación consolidado PT1 Salud, Sexualidad y Género, de la Consellería de Cultura, Educación e Ordenación Universitaria. Siendo las líneas principales de investigación: comportamientos sexuales y adictivos de jóvenes, salud y calidad de vida.

María D. Dapía Conde, Titular de Universidad en el área de Teoría e Historia de la Educación (Departamento de Análisis e Intervención Psicopedagogía) de la Universidad de Vigo, impartiendo docencia fundamentalmente en la Titulación de Educación Social. Miembro del grupo de investigación, «Educación y Salud» GIES-10, siendo su línea de trabajo Ocio, Salud y Calidad de Vida, con aportaciones en Congresos, libros y artículos científicos.

Alberto Alonso Álvarez, Licenciado con Grado en Psicopedagogía y alumno predoctoral de la Universidad de Vigo, dentro del Departamento de Análisis e Intervención Psicosocioeducativa. Actualmente realizando Tesis doctoral en el ámbito de menores en sistemas de protección y reforma. Investigador asociado del grupo PTI investigación PT1 Salud, Sexualidad y Género.

Elizier Pazos Millán, Licenciado en Psicopedagogía y profesor de primaria. Realizando Tesis Doctoral sobre adolescentes y consumo de sustancias tóxicas. Investigador asociado del grupo PTI investigación PT1 Salud, Sexualidad y Género.

Dirección de los Autores: Departamento Análisis e Intervención

Psicosocioeducativa

Universidad Vigo

Facultad de Ciencias de la Educación 32004 - Ourense

E-mail: jfailde@uvigo.es

ddapia@uvigo.es

alalonso@uvigo.es

elizierpazos@aol.es

Fecha Recepción del Artículo: 09. Mayo. 2013

Fecha Modificación Artículo: 30. Diciembre. 2013

Fecha Aceptación del Artículo: 06. Febrero. 2014

Fecha Revisión para publicación: 09. Enero. 2015 